\title{
Classificação de tomate-cereja em função do tamanho e peso dos frutos
}

\author{
Carolina Fernandes; José Eduardo Corá; Leila T. Braz \\ UNESP-FCAV, Via de Acesso Prof. Paulo Donato Castellane, s/n, 14884-900 Jaboticabal-SP; carol@fcav.unesp.br; cora@fcav.unesp.br; \\ leilatb@fcav.unesp.br
}

\begin{abstract}
RESUMO
O objetivo deste trabalho foi propor classes de tamanho e peso para frutos de tomate-cereja. O experimento foi conduzido em Jaboticabal, São Paulo. Foram utilizados frutos da cultivar Sindy, provenientes de 60 plantas cultivadas em casa-de-vegetação. Para classificação dos frutos, desenvolveu-se um método no qual foram utilizadas cinco caixas plásticas sobrepostas. Quatro caixas tiveram suas bases perfuradas, com orifícios de diâmetro igual a 20;25; 30 e $35 \mathrm{~mm}$. As quatro caixas foram sobrepostas, adicionando-se uma quinta caixa, sem orifícios, utilizada sob as demais, para coletar os frutos que passassem pelos orifícios das caixas superiores. Os frutos retidos em cada uma das caixas foram contados e pesados. Com esses dados, calculou-se o peso médio dos frutos para cada classe de tamanho. A frequiência de frutos em cada classe variou ao longo do período de colheita. A porcentagem de frutos produzidos durante o período de colheita para as diferentes classes foi de 5\% para gigante, $22 \%$ para grande, $42 \%$ para médio, $20 \%$ para pequeno e $11 \%$ para descarte. Os valores de peso (P) e diâmetro (D) dos frutos correlacionaram-se linearmente, seguindo equação ajustada $(\mathrm{P}=1,05$ $\mathrm{D}-14,71)$ com coeficiente de correlação $\left(\mathrm{R}^{* *}=0,99, \mathrm{p}<0,01\right)$. Utilizando o sistema descrito, foi possível propor uma classificação para tomate-cereja associando-se o diâmetro e o peso dos frutos. O método proposto mostrou-se viável pela praticidade e simplicidade.
\end{abstract}

Palavras-chave: Lycopersicon esculentum, normas de classificação, pós-colheita.

\begin{abstract}
Grading of cherry tomatoes based on fruit size and weight

The purpose of this experiment was to propose a grading system for cherry tomatoes based on fruit size and weight. The experiment was carried out at Jaboticabal, São Paulo State, Brazil. Fruits of cherry tomatoes, cultivar Sindy, harvested out of 60 plants grown in a greenhouse were evaluated. To separate fruits of different sizes, a five plastic box system developed. In this system, the bottom of four boxes drilled with holes of $20 ; 25 ; 30$, and $35 \mathrm{~mm}$ of diameter. The fifth box had no holes and was used as a collector box. Fruits retained in each of the boxes were counted and weighed. The average weight of the fruits was calculated for each class size. Fruit frequency in each class varied during the harvest period. The percentage of fruits was as follows: $5 \%$ of giant fruits, $22 \%$ of large fruits, $42 \%$ of medium fruits, $20 \%$ for small fruits, and $11 \%$ of discard. There was a linear correlation between the fruit weight (W) and diameter (D) (W = $\left.1.05 \mathrm{D}-14.71, \mathrm{R}^{* *}=0.99, \mathrm{p}<0.01\right)$. The system used was efficient for grading cherry tomatoes by means of associating fruit diameter and weight. In addition, it was practical and simple.
\end{abstract}

Keywords: Lycopersicon esculentum, grading, post-harvest.

\section{(Recebido para publicação em 18 de maio de 2006; aceito em 16 de abril de 2007)}

$\mathrm{O}$ Programa Brasileiro para a Modernização da Horticultura surgiu como resposta aos principais problemas das cadeias de produção de frutas e hortaliças, entre eles a inexistência de padrões mensuráveis de qualidade (CEAGESP, 2004). Como resultado deste programa, atualmente, estão disponíveis normas de classificação de diversas hortaliças, incluindo o tomate. A adoção voluntária às normas de classificação do tomate é o caminho que leva à transparência nas operações comerciais, beneficiando produtores, distribuidores, varejistas e consumidores. A classificação é a comparação do produto com padrões pré-estabelecidos. O julgamento obtido dessa comparação permite fazer o enquadramento do produto em grupo, subgrupo, classe, calibre e tipo, tornando possível uma interpretação única. Um produto classificado é um produto separado por tamanho, cor e qualidade, de modo a se obter lotes homogêneos e caracterizados de maneira clara e mensurável (CEAGESP, 2004).

$\mathrm{O}$ tomate é classificado em relação à coloração do fruto, quanto ao tipo ou categoria e quanto ao tamanho do fruto (CEAGESP, 2004). A coloração do fruto varia em função do seu estádio de maturação. Para este atributo, o tomate é classificado em cinco subgrupos: verde, salada, colorido, vermelho e molho. Já o tipo ou categoria refere-se à ocorrência de defeitos graves e leves na amostra. Para este atributo, o tomate é classificado em extra, tipo I, tipo II e tipo III. Para tamanho do fruto, o tomate é classificado em dois grupos: oblongo, quando o diâmetro longitudinal é maior que o diâmetro transversal, e redondo, quando o diâmetro longitudinal é menor ou igual ao transversal. De acordo com o maior diâmetro transversal do fruto, o tomate do grupo oblongo é classificado em três classes: grande
( $>60 \mathrm{~mm})$, médio $(50<\varnothing<60 \mathrm{~mm})$ e pequeno $(40<\varnothing<50 \mathrm{~mm})$. De acordo com o maior diâmetro transversal do fruto, o tomate do grupo redondo, com exceção do tomate-cereja, é classificado em quatro classes: gigante $(\varnothing>100 \mathrm{~mm})$, grande $(90<\varnothing<100 \mathrm{~mm})$, médio $(65<$ $\varnothing<90 \mathrm{~mm})$ e pequeno $(50<\varnothing<65 \mathrm{~mm})$. Para complementar a classe, adiciona-se o calibre (CEAGESP, 2004).

Pelos atributos de classificação apresentados, fica evidente que o tomatecereja não se enquadra nas normas de classificação apenas para as classes de tamanho. Os valores considerados para o diâmetro transversal dos frutos de tomate são sempre maiores que aqueles observados no tomate-cereja.

O objetivo deste trabalho foi propor classes de tamanho e peso para frutos de tomate-cereja. Para tanto, será avaliada a existência de correlação entre essas duas características. 


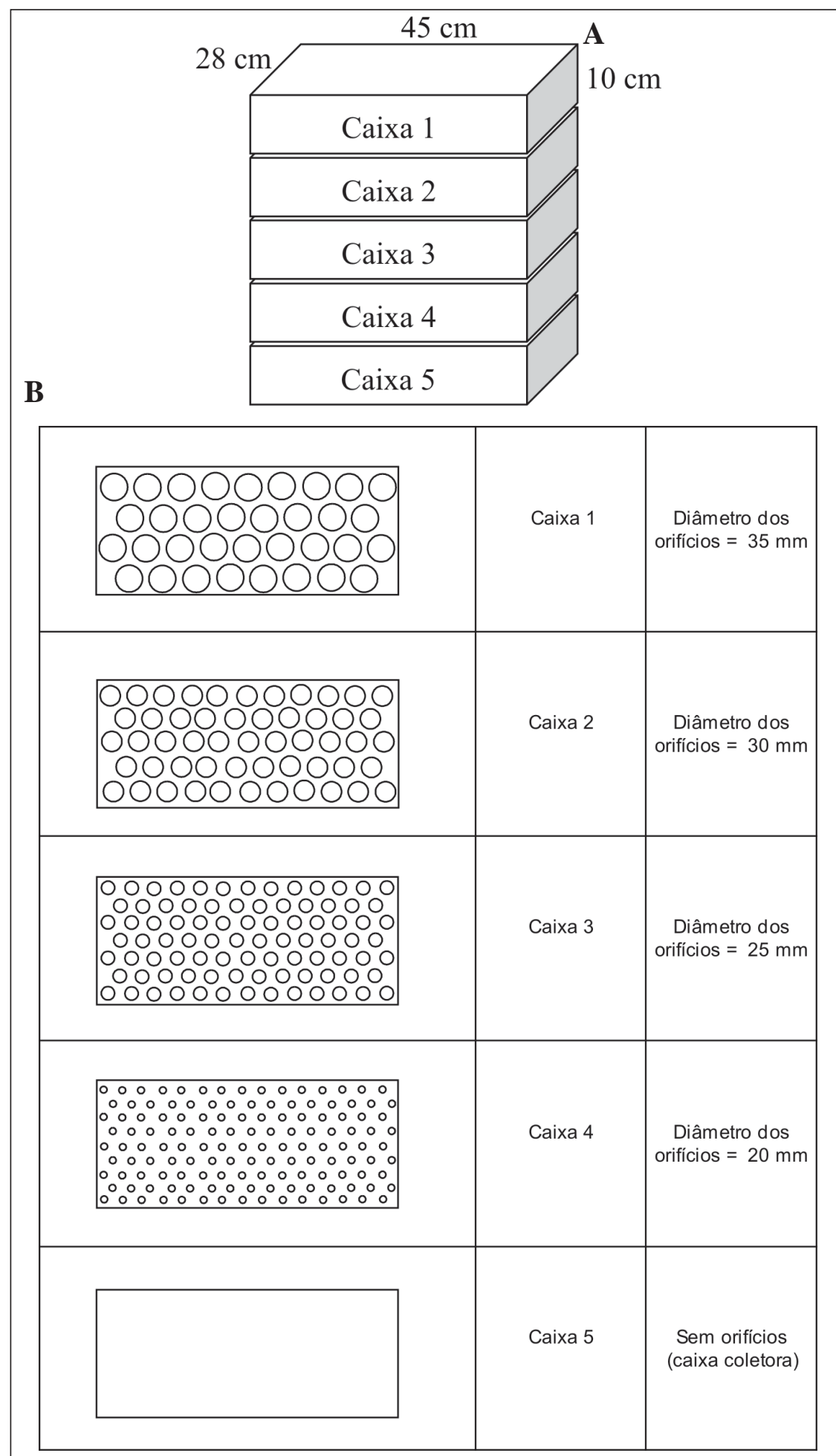

Figura 1. Esquema representativo das caixas utilizadas para a separação de tomate-cereja em diferentes classes de tamanho. A) conjunto das cinco caixas sobrepostas; B) caixas individuais mostrando a distribuição dos orifícios nas bases (Representative scheme of the system used for grading cherry tomatoes in different size classes. A) aspect of the five boxes; B) each box is shown individually, with detail of the bottom drills). Jaboticabal, UNESP-FCAV, 2004.

\section{MATERIAL E MÉTODOS}

$\mathrm{O}$ experimento foi conduzido em casa-de-vegetação da UNESP-FCAV, em Jaboticabal, SP $\left(21^{\circ} 14^{\prime} 05^{\prime \prime S}\right.$, $48^{\circ} 17^{\prime} 09^{\prime \prime} \mathrm{W}$, altitude de $\left.614 \mathrm{~m}\right)$. O clima, segundo a classificação de Köppen, é do tipo Aw com transição para Cwa
(Volpe, 2004. Comunicação pessoal). A temperatura e a umidade relativa do ar no interior da casa de vegetação foram obtidas por meio de um termohigrógrafo com registro contínuo dos valores. Durante o ciclo da cultura, as médias mensais de temperatura máxima diária, nos meses de julho, agosto, setembro, outubro e novembro foram, respectivamente, $33 ; 33 ; 35 ; 36$ e $38^{\circ} \mathrm{C}$. As médias mensais de temperatura mínima diária foram $11 ; 11 ; 13 ; 14$ e $16^{\circ} \mathrm{C}$, nos mesmos meses. As médias mensais de umidade relativa do ar máxima diária foram 98; 98; 96; 95 e 94\%, enquanto as médias mensais da umidade relativa do ar mínima diária foram 40; 40; 39; 38 e $37 \%$, também nos mesmos meses.

O cultivo do tomateiro cultivar Sindy, híbrido $F_{1}$ do tipo cereja, foi conduzido em substrato com a utilização de fertirrigação. $\mathrm{O}$ substrato foi composto por areia média, bagaço de cana-de-açúcar e casca de amendoim. Para o fornecimento de nutrientes, utilizou-se a solução nutritiva recomendada por Moraes (1997) para o cultivo do tomateiro em sistema NFT (Técnica do Fluxo Laminar de Nutrientes).

As mudas do tomateiro foram transplantadas no estádio de quatro folhas definitivas. Foram utilizados vasos de plástico, com capacidade para $5 \mathrm{~L}$. Conduziu-se uma planta por vaso, com duas hastes por planta, no espaçamento de 1,0 $\mathrm{m} \times 0,5 \mathrm{~m}\left(2\right.$ plantas $\left.\mathrm{m}^{-2}\right)$, totalizando 60 plantas, que foram dispostas inteiramente ao acaso. A desbrota e a condução vertical da cultura foram realizadas semanalmente. A poda dos ponteiros foi realizada quando as plantas atingiram dois metros de altura e apresentavam de sete a oito cachos por haste. Para o controle de pragas e doenças foram realizadas, conforme a necessidade, pulverizações com defensivos nas doses e épocas recomendadas pelo fabricante para o tomateiro. O período de colheita iniciou-se 78 dias após o transplantio e teve duração de 57 dias. A colheita dos frutos foi realizada duas vezes por semana.

Para o processo de separação dos frutos de tomate-cereja por tamanho e peso, foi desenvolvido um método no qual foram utilizadas cinco caixas plásticas, com $45 \mathrm{~cm}$ de comprimento, $28 \mathrm{~cm}$ de largura e $10 \mathrm{~cm}$ de altura (Figura 1). Quatro 
caixas tiveram suas bases perfuradas, com orifícios de 20; 25; 30 e $35 \mathrm{~mm}$ de diâmetro, de acordo com a variação do diâmetro dos frutos observada em cultivos anteriores. As caixas foram sobrepostas em ordem decrescente do diâmetro dos orifícios, de cima para baixo. Uma quinta caixa sem orifícios foi utilizada sob as demais, para coletar os frutos que passassem pelos orifícios das quatro caixas anteriores. Durante o período de colheita, os frutos colhidos foram depositados na caixa superior e, em seguida, o conjunto de cinco caixas plásticas foi agitado, manualmente, até os frutos passarem ou ficarem retidos nas diferentes caixas. Os frutos que ficaram retidos na caixa coletora foram descartados, em virtude do seu tamanho diminuto. Os frutos que ficaram retidos em cada uma das quatro caixas superiores foram pesados e contados. Posteriormente, com esses dados, calculou-se o peso médio dos frutos para cada classe de tamanho. Foram colhidos todos os frutos produzidos pelas plantas em avaliação. Considerou-se como uma repetição a colheita dos frutos provenientes de duas plantas. O número de frutos por repetição foi variável em função da quantidade de frutos colhidos, tendo como valor mínimo um fruto e valor máximo 167 frutos, com média de 69 frutos. Ao longo do período de colheita, esse processo de separação de frutos foi repetido por 360 vezes.

A correlação entre peso e diâmetro do fruto foi avaliada pela análise de regressão linear. O diâmetro dos frutos baseou-se nos frutos retidos em cada caixa.

\section{RESULTADOS E DISCUSSÃO}

A frequiência de frutos em cada classe variou ao longo do período de colheita (Figura 2). As plantas produziram frutos da classe gigante nos quinze dias iniciais do período de colheita. Os frutos grandes, médios e pequenos foram obtidos em praticamente todo o período de colheita. Praticamente todos os frutos descartados foram obtidos na última semana da colheita. Considerouse que as quatro classes de tamanho dos frutos obtidas são necessárias, ainda que a classe de frutos gigantes tenha

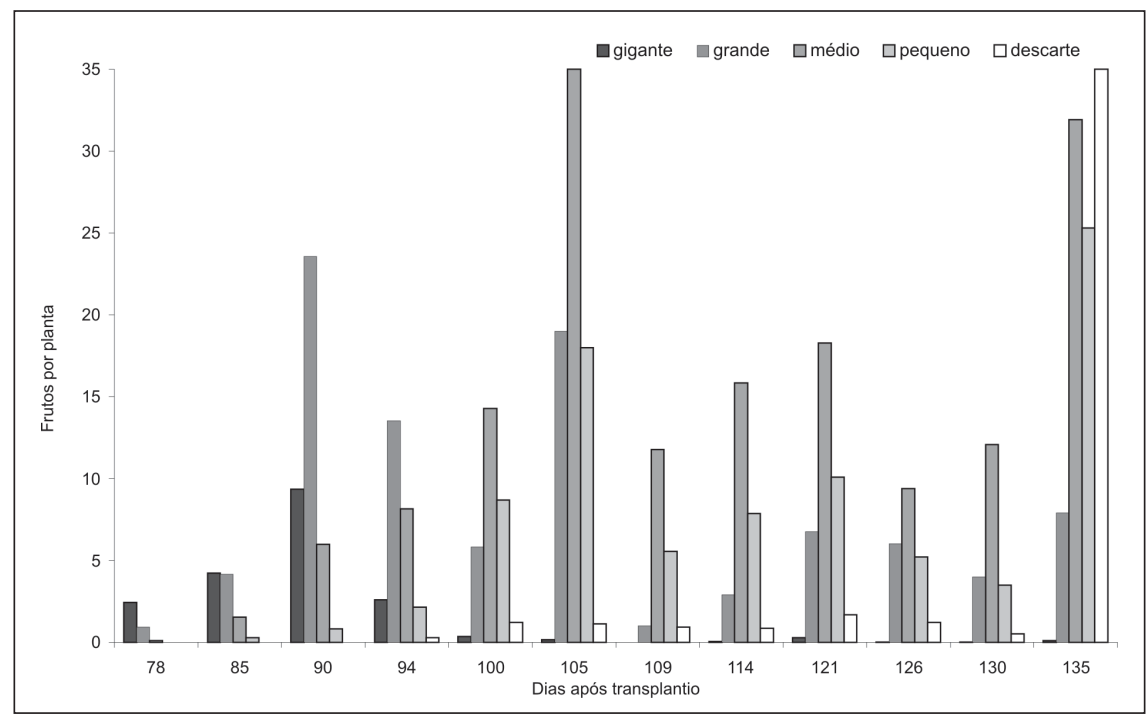

Figura 2. Número médio de frutos de tomate-cereja por planta em cada classe de tamanho, ao longo do período de colheita (Average number of cherry tomatoes per plant in each size class during the harvest period). Jaboticabal, UNESP-FCAV, 2004.

Tabela 1. Número total e porcentagem de frutos colhidos para cada classe de tomate-cereja, provenientes das 60 plantas avaliadas (Total and percentual of harvested fruits in each class of cherry tomato, evaluated of 60 plants). Jaboticabal, UNESP-FCAV, 2004.

\begin{tabular}{ccc}
\hline Classes & Número de frutos & $\%$ \\
\hline gigante & 1177 & 05 \\
grande & 5388 & 22 \\
médio & 10504 & 42 \\
pequeno & 4971 & 20 \\
descarte & 2711 & 11 \\
Total & 24751 & \\
\hline
\end{tabular}

Tabela 2. Classificação de tomate-cereja em classes e calibres (Cherry tomatoes grading based in classes and diameter). Jaboticabal, UNESP-FCAV, 2004.

\begin{tabular}{cccc}
\hline Classes & Calibres & $\begin{array}{c}\text { Maior diâmetro } \\
\text { transversal do fruto } \\
(\mathbf{m m})\end{array}$ & Peso do fruto $(\mathbf{g})$ \\
\hline gigante & $3 \mathrm{Gi}$ & maior que 35 & maior que 20 \\
grande & $3 \mathrm{Gr}$ & maior que 30 até 35 & entre 15 e 20 \\
médio & $2 \mathrm{M}$ & maior que 25 até 30 & entre 10 e 15 \\
pequeno & $2 \mathrm{P}$ & maior que 20 até 25 & entre 5 e 10 \\
\hline
\end{tabular}

correspondido a apenas $5 \%$ do total de frutos colhidos (Tabela 1). Acredita-se que essa classe de tamanho atenda a um nicho diferenciado de consumidores, alcançando assim maior valor de mercado, devido principalmente à aparência. A porcentagem de frutos descartados foi de $11 \%$, sendo estes pequenos demais para o comércio.

Os valores de peso (P) e diâmetro (D) dos frutos correlacionaram-se linearmente, seguindo uma equação ajustada $(\mathrm{P}=1,05 \mathrm{D}-14,71)$, com coefi- ciente de correlação $\left(\mathrm{R}^{* *}=0,99\right.$, $\mathrm{p}<0,01)$. A existência dessa correspondência funcional confirma a dependência das variáveis avaliadas (Banzatto \& Kronka, 1989; Gomes, 2000). Portanto, há correlação positiva entre o diâmetro e o peso do fruto para tomate-cereja. Isto significa que o tomate-cereja pode ser classificado por diâmetro ou peso, não havendo necessidade de utilizar os dois parâmetros de classificação.

Acompanhando as características do sistema de classificação proposto por 
CEAGESP (2004) para tomate e baseando-se nos resultados obtidos no presente estudo, sugere-se que o tomatecereja seja classificado nas quatro classes de diâmetro propostas e quatro calibres (Tabela 2). Acredita-se que o sistema proposto possa ser utilizado para outras cultivares de tomate-cereja, uma vez que a variação de tamanho será detectada dentro de cada classe. Entretanto, ressalta-se a necessidade de confirmar essa informação por meio de outros experimentos que avaliem o siste- ma de classificação aqui proposto para outras cultivares de tomate-cereja.

Concluindo, pode-se propor a classificação para tomate-cereja associando-se o diâmetro e o peso dos frutos. Além disso, o método proposto é viável pela praticidade e simplicidade.

\section{AGRADECIMENTOS}

Ao CNPq, pela concessão da bolsa de doutorado.

\section{REFERÊNCIAS}

BANZATTO DA; KRONKA SN. 1989. Experimentação agrícola. Jaboticabal: FUNEP. 247p.

CEAGESP. Classificação do tomate. 2004, 16 de outubro. Disponível em www.ceagesp.com.br.

GOMES FP. 2000. Curso de estatística experimental. Piracicaba: Degaspari. 477p.

MORAES CAG. 1997. Hidroponia: como cultivar tomates em sistema NFT (técnica do fluxo laminar de nutrientes). Jundiaí: DISQ Editora. $141 \mathrm{p}$. 